\title{
Development of the Transdermal Potential of Human Skin
}

\author{
P. M. MACKAY, M. R. MILLAR, M. I. LEVENE, C. M. HOY, S. F. DEALLER, M. PAPOULI, \\ AND P. C. NG \\ Departments of Paediatrics [P.M.M., M.I.L., M.P., P.C.N.] and Microbiology [M.R.M., C.M.H., S.F.D.], The \\ General Infirmary at Leeds, Leeds, West Yorkshire, LS2 9NS, England
}

\begin{abstract}
The development of the transdermal electrical potential (TDP) with postnatal age was studied in neonates born at gestational ages of 25 to 42 wk. The TDP of neonates born at less than 28 wk gestational age was of similar magnitude over the whole skin surface when measured in the first $5 \mathrm{~d}$ of life (mean value $-5.4 \mathrm{mV}$; skin surface negative with respect to s.c. tissues). The TDP increased progressively with increasing gestational and postnatal age. The rate of increase of the TDP with postmenstrual age was not accelerated in neonates born prematurely. TDP values of infants born at term were lower than those of adults, but the sites of high and low TDP were similar in both term infants and adults. (Pediatr Res 29: 78-81, 1991)
\end{abstract}

\section{Abbreviations}

TDP, transdermal electrical potential

An electrical potential difference exists across many epithelia. It results from the balance of active transport of ions across the epithelium and the permeability of the epithelium to the passive diffusion of ions along the electrochemical gradients produced by the active transport processes. The magnitude of the potential is dependent on the structure and function of the epithelium and may be altered in disease states, as in cystic fibrosis, where epithelial cells have decreased passive permeability to chloride ions. This causes decreased reabsorption of chloride from sweat, resulting in increased negativity of the transdermal potential and abnormally high sweat chloride concentration.

In adult humans, the TDP is generated largely in the sweat glands. Its magnitude depends on the activity and density of distribution of the glands, the hydration of the epidermis, and the integrity and permeability of the living cell layers of the epidermis (1). All of these factors are altered in premature infants.

We studied the evolution of the TDP in premature infants compared to that of term infants and of adults.

\section{SUBJECTS AND METHODS}

The TDP was measured in three groups of subjects over a 3month period.

Group 1. One hundred and nineteen sets of observations of TDP were obtained from $49(61 \%)$ of 71 neonates of less than 37 wk gestational age admitted to the Peter Congdon Neonatal Unit in Leeds. Each set of observations consisted of readings

Received February 23, 1990; accepted August 8, 1990.

Correspondence and reprint requests: Dr. P. M. Mackay, Department of Paediatrics, Clarendon Wing, The General Infirmary at Leeds, Leeds, West Yorkshire LS2 9NS, England.

Supported by Viggo, Faraday Road, Dorcan, Swindon, Wiltshire, SN3 $5 \mathrm{HZ}$. from the dorsum of the hand, the flexor and extensor aspects of the forearm, the chest and the back (when the baby was able to be turned safely), the dorsum of the foot, and the middle of the plantar surface of the foot.

The results were grouped into 5-d periods of postnatal age. When more than one set of observations was collected during one time period from one individual, only the result nearest the median day of the time period was considered. This resulted in analysis of 86 sets of observations from 49 premature infants.

All infants required i.v. cannulas for their medical management. Thirty-three $(67.3 \%)$ of the 49 infants had respiratory distress syndrome.

Group 2. Nine sets of observations from nine term infants aged 0-5 d were analyzed. Each set of observations consisted of TDP measurements from 30 skin sites, which were obtained while the infants were asleep.

All of the infants were asymptomatic at the time of observation but were receiving antibiotics through peripheral i.v. cannulas for suspected perinatal infection. No pathogens were cultured from pretreatment blood cultures in this group.

Group 3. One set of observations of TDP from 30 skin sites was obtained from each of 11 healthy adult volunteers aged 28$45 \mathrm{y}$ of whom seven were male and four female.

Measurement of TDP. The potential difference between the lumen of a superficial vein and the skin surface at multiple sites was measured using silver/silver chloride electrodes connected to a high impedance amplifier (input impedance $>10^{12} \mathrm{ohms}$ ) in series with a digital voltmeter (J. Fluke Manufacturing Co., Inc.).

The electrodes were presoaked in $0.9 \%$ sodium chloride for $1-$ $4 \mathrm{~h}$ before use. The reference electrode was connected to the venous lumen of the patient using a Teflon i.v. cannula and polyethylene tubing filled with $0.9 \%$ sodium chloride. The measuring electrode was connected to the skin using a $0.9 \%$ sodium chloride $/ 0.75 \%$ agar bridge contained in polyethylene tubing. The skin contact area was $0.8 \mathrm{~cm}^{2}$.

Electrode imbalance was measured by short-circuiting the electrodes across a $0.9 \%$ sodium chloride bridge before and after each observation and the value subtracted from the observation to give the correct value for the TDP. Observations were repeated if the electrode imbalance was $> \pm 5 \mathrm{mV}$ or its variation over the period of the observation was $> \pm 0.5 \mathrm{mV}$. Continuity of electrical contact between the measuring apparatus and the patient was tested by measurement of the circuit resistance before each set of observations.

The TDP was recorded after washing the skin with $0.9 \%$ sodium chloride and wiping dry with cotton wool. The $0.9 \%$ sodium chloride $/ 0.75 \%$ agar bridge was applied directly to the skin until a stable reading was obtained. This was defined as variation of less than $0.3 \mathrm{mV}$ over $15 \mathrm{~s}$ and usually occurred 30 $\mathrm{s}$ to $2 \mathrm{~min}$ after application of the skin bridge. Care was taken not to abrade or injure the skin as these caused the TDP to decrease.

Measurement of liquid junction potential. The liquid junction 
potential between fresh heparinized blood samples $(10 \mathrm{U} / \mathrm{mL}$ of haparin) and $0.9 \%$ sodium chloride was measured in vitro at $37^{\circ} \mathrm{C}$ using silver/silver chloride electrodes with flowing $3 \mathrm{M}$ potassium chloride as a reference bridge and flowing $0.9 \%$ sodium chloride as the measuring bridge.

Estimation of reproducibility of measurements. Eleven consecutive measurements of a standard signal of $16.1 \mathrm{mV}$ were performed using a different set of measuring electrodes and connecting bridges for each measurement.

Eleven consecutive measurements of TDP at the same site in a single subject were performed using a different set of measuring electrodes and connecting bridges for each measurement.

Coefficients of variation were calculated for the reproducibility of a standard signal and for the variation of TDP in a stable subject over time.

Analysis of results. The infants were grouped according to gestational age at birth as follows: $25-27,28-30,31-33,34-36$, and $37-42 \mathrm{wk}$. The mean gestational age for each group was used in plotting Figure 1. The postmenstrual age was calculated by adding the postnatal age to the gestational age at birth (Fig. 2.)

The $t$ test was used for the calculation of $p$ values.

Ethical approval. Local Ethical Committee approval was obtained for the study and patient or parental consent was obtained for each individual. It was considered unethical to insert i.v. cannulas into babies for the sole purpose of obtaining TDP measurements. Thus it was not possible to obtain measurements from a group of unequivocally normal infants.

\section{RESULTS}

In all cases, the skin surface had a negative voltage with respect to the underlying tissues. Figure 1 illustrates that the TDP of babies aged less than $5 \mathrm{~d}$ born at 25-27 wk gestation was low at all sites and there was no statistically significant difference between the skin sites of highest and lowest TDP $(0.05<p<0.1)$. The mean TDP for all sites of this group was $-5.4 \mathrm{mV}$. The TDP increased at all sites with increasing gestational age. By 36 $w k$, the TDP values were similar to those at term. The TDP at every site was higher in infants at term than in infants at 25-27 wk gestational age ( $p<0.001$ for each site, Table 1). The

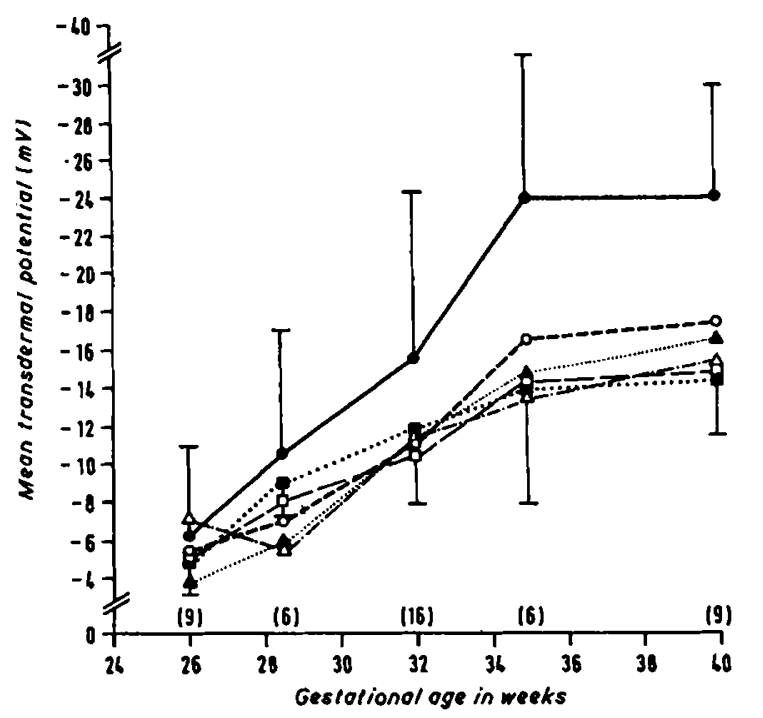

Fig. 1. Variation of TDP, measured at various sites, with gestational ige (at postnatal age $0-5 \mathrm{~d}$ ). $\bullet$, foot-plantar surface; $\boldsymbol{\Lambda}$, foot-dorsum; $\boldsymbol{\square}$, sack (over T4); $O$, forearm flexor; $\square$, forearm extensor; $\Delta$, chest over iternum. Parentheses, $n$ for the calculation of each data point; vertical jars, $1 \mathrm{SD}$.

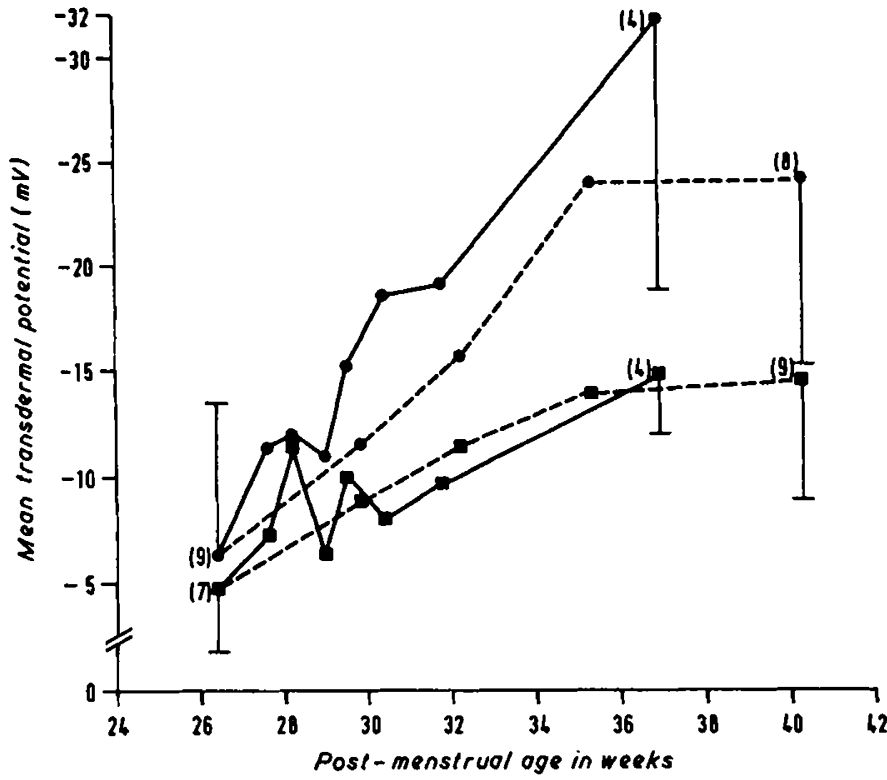

Fig. 2. The development of the TDP with postmenstrual age in babies born at 25-27 wk (solid lines) compared with values measured at 0-5 d of age in babies born at varying gestations (broken lines). $\bullet$, plantar surface of foot, $\mathbf{\square}$, back (over T4). Vertical hars indicate $\mathrm{SD}(n)$.

differentiation of the skin into areas of high and low TDP was well established at term. Several areas of low TDP had values approaching those of adults, whereas the areas of high TDP still had considerably lower values in term infants than in adults, as shown in Table 1.

The sites of highest TDP were the palms of the hands and soles of the feet; the sites of lowest TDP were on the scalp in both term infants and adults. The correlation coefficient between the mean TDP of term infants and adults was 0.69 .

The development of the TDP in relation to postmenstrual age is shown in Figure 2. The rate of increase of TDP at sites of high and intermediate values was similar in neonates born at 25-27 wk and neonates born at greater gestations, showing that premature birth did not accelerate the development of the TDP.

In Figure 2, the last points for TDP values of the 25-27 wk gestation group did not contain data from all of the initial cohort due to discharge of patients from the unit and one death, which occurred at postnatal age $3 \mathrm{~d}$. Thus, data loss due to mortality exerted little influence on the overall distribution of results.

The coefficient of variation of measurement of a standard signal of $16.1 \mathrm{mV}$ was $2 \%$.

The coefficient of variation of TDP in a single stable subject over a period of $3 \mathrm{~h}$ was $17 \%$.

The mean liquid junction potential between blood and $0.9 \%$ saline measured in vitro at $37^{\circ} \mathrm{C}$ was $-2.6 \mathrm{mV}$, with a range of -2.3 to $-2.9 \mathrm{mV}$ in six samples.

\section{DISCUSSION}

Our study examined the development of the TDP in premature neonates and compared neonatal values with those of adults.

The TDP in adults derives largely from the sweat glands (1), of which both the secretory (2) and reabsorptive (3) elements have electrogenic properties. The living epithelium between the sweat glands has a smaller intrinsic electrogenic potential. These potentials are generated by the active transport of ions across epithelial cells and are partly dissipated by passive diffusion of ions along the resulting transepithelial electrical gradient. The TDP measured at the skin surface is the sum of these effects and may additionally be influenced by the degree of hydration of the stratum corneum, which when wet may dissipate the potential at the orifice of the sweat ducts into the surrounding epithelium (1). 
Table 1. Transdermal electrical potentials (negative) at various sites in preterm and term infants at 0-5 days of age and in adults.

\begin{tabular}{|c|c|c|c|c|c|c|c|c|c|c|}
\hline \multirow[b]{3}{*}{ Site } & \multicolumn{10}{|c|}{ Gestational age (wk) } \\
\hline & \multicolumn{3}{|c|}{$25-27$} & \multicolumn{3}{|c|}{$37-42$} & \multicolumn{3}{|c|}{ Adults } & \multirow[b]{2}{*}{$p$-value* } \\
\hline & Mean $(\mathrm{mV})$ & SD & $n$ & Mean $(\mathrm{mV})$ & $\mathrm{SD}$ & $n$ & Mean $(\mathrm{mV})$ & SD & $n$ & \\
\hline Thumb & & & & 24.0 & 2.2 & 9 & 34.2 & 6.1 & 11 & + \\
\hline Foot plantar & 6.1 & 5.0 & 9 & 23.9 & 6.1 & 8 & 42.8 & 3.2 & 7 & $x+$ \\
\hline Middle finger & & & & 23.8 & 4.6 & 9 & 38.1 & 5.3 & 11 & + \\
\hline Great toe & & & & 22.1 & 4.6 & 9 & 38.9 & 6.5 & 11 & + \\
\hline Calf & & & & 17.5 & 3.8 & 9 & 17.6 & 5.3 & 10 & \\
\hline Forearm flexor & 5.3 & 2.2 & 9 & 17.3 & 4.1 & 9 & 18.9 & 5.6 & 11 & $\mathrm{x}$ \\
\hline Suprapatellar & & & & 17.2 & 4.2 & 8 & 20.3 & 5.4 & 11 & \\
\hline Supraumbilical & & & & 17.0 & 6.6 & 9 & 19.3 & 9.8 & 11 & \\
\hline Heel & & & & 17.0 & 10.0 & 9 & 44.3 & 6.4 & 11 & + \\
\hline Nipple & & & & 16.6 & 3.6 & 8 & 20.2 & 5.7 & 11 & \\
\hline Back (@L4) & & & & 16.5 & 3.1 & 9 & 19.1 & 9.9 & 11 & \\
\hline Foot, dorsum & 3.7 & 3.6 & 9 & 16.5 & 4.5 & 9 & 29.0 & 7.4 & 11 & $x+$ \\
\hline Shin & & & & 16.2 & 3.3 & 9 & 16.6 & 3.4 & 11 & \\
\hline Loin & & & & 16.2 & 2.6 & 8 & 15.5 & 5.2 & 9 & \\
\hline Shoulder, tip & & & & 15.7 & 3.6 & 9 & 18.0 & 6.8 & 7 & \\
\hline Biceps & & & & 15.6 & 3.9 & 7 & 15.8 & 5.9 & 11 & \\
\hline Sternum & 7.1 & 2.1 & 7 & 15.2 & 4.1 & 9 & 20.6 & 7.7 & 11 & $\mathrm{x}$ \\
\hline Trapezius, upper border & & & & 14.7 & 5.3 & 9 & 18.8 & 6.6 & 10 & \\
\hline Forearm, extensor & 5.2 & 1.8 & 9 & 14.5 & 3.2 & 9 & 16.9 & 4.3 & 11 & $\mathbf{x}$ \\
\hline Back (@T4) & 4.8 & 2.1 & 7 & 14.2 & 4.0 & 9 & 21.9 & 9.0 & 11 & $x+$ \\
\hline Hand, dorsum & 5.9 & 2.9 & 9 & 13.9 & 7.1 & 9 & 22.2 & 5.7 & 11 & $x+$ \\
\hline Popliteal fossa & & & & 13.6 & 6.3 & 9 & 17.6 & 5.8 & 10 & \\
\hline Chin & & & & 13.3 & 3.2 & 9 & 21.0 & 5.3 & 11 & + \\
\hline Cheek & & & & 13.1 & 3.9 & 8 & 17.6 & 6.1 & 7 & \\
\hline Occiput & & & & 12.8 & 5.6 & 9 & 14.4 & 6.7 & 11 & \\
\hline Mastoid & & & & 12.6 & 4.4 & 9 & 17.6 & 7.0 & 11 & \\
\hline Forehead & & & & 11.8 & 5.1 & 9 & 18.8 & 5.9 & 11 & + \\
\hline Upper lip & & & & 11.5 & 3.9 & 9 & 20.6 & 6.7 & 11 & + \\
\hline Vertex & & & & 11.2 & 3.5 & 9 & 16.0 & 6.0 & 11 & \\
\hline Nose & & & & 9.1 & 5.6 & 9 & 22.7 & 5.3 & 11 & + \\
\hline
\end{tabular}

${ }^{*} \mathrm{x}=$ Significant difference between the values at $25-27$ wk gestation and at 37-42 wk gestation $(p<0.001) ;+=$ significant difference between values at $37-42 \mathrm{wk}$ gestation and adult values $(p<0.05)$.

We observed that the TDP in premature neonates was very different from that of adults, reflecting the profound differences of skin structure and function between immature neonates and adults. The number of sweat glands in the fetus has reached the adult level by the end of the 2nd trimester, at which time the sweat ducts are patent (4) and the distribution of the glands is of the adult pattern, being in higher density on the palms and soles and lower on the trunk. Differences in TDP between areas with different densities of sweat glands would be expected, if the glands at this gestation showed electrogenic activity. The observation that the TDP was of similar magnitude at all sites in infants of less than 28 wk gestation suggests that the sweat glands at this age have no electrogenic activity as a consequence of functional immaturity. The gradual increase in TDP and increasing differentiation between sites of higher and lower TDP reflects increasing functional maturity of the sweat glands until 36 wk gestation, when the values are similar to those seen at term.

The rate of development of the TDP was not modified by premature birth (Fig. 2). This contrasts with the development of thermal sweating ability in premature infants, which is markedly accelerated by premature birth (5) and occurs over most of the body surface except the palms and soles. The gradual development of the TDP on the sole of the foot also contrasts to the abrupt development of emotional sweating reported to occur on the palms and soles at $36 \mathrm{wk}$ postmenstrual age (6).

The disparity between the development of electrogenic activity and the development of active sweating is consistent with the hypothesis that as progressive maturation of the secretory and reabsorptive portions of the sweat gland occurs, their electrogenic activity increases. However, as long as reabsorptive activity ex- ceeds secretory activity, no active sweating will be measured at the skin surface. Thus, measurement of the TDP may be a more sensitive and convenient indicator of the early functional development of the sweat gland than is the measurement of stressinduced sweating.

The TDP in the most premature infants was similar at all sites. With increasing postmenstrual age, there was increasing differentiation of the skin into areas of high and low TDP. Sites of high and low TDP values were broadly similar in term infants and adults, indicating that the distribution of functioning sweat glands is of the adult pattern. The generally lower TDP despite the higher concentration of sweat glands per unit area of term infants (7) may reflect functional immaturity of the glands or, alternatively, may reflect increased skin permeability allowing dissipation of the transepithelial potential by passive diffusion of ions through the skin.

The method used in TDP measurement of all patients was identical, so it is of interest that although the SD of observations on healthy adults were similar to those on asymptomatic term infants, the SD on premature infants were larger in proportion to their means even when corrected for changes with gestational age, suggesting that the TDP may be affected by either the patient's pathology or the treatment received. The spectrum of illness and treatment in our babies was typical of that in a regional medical neonatal unit. One factor that caused abnormally low TDP readings was damage to the skin at the site of measurement, which effectively dissipated the TDP in the surrounding area. This effect was seen in the lower values of means and larger SD of readings from the heels and dorsa of the hands of term babies when compared with adults. These were the sites 
that were most frequently subjected to iatrogenic skin trauma in our neonatal population. A similar observation has been made on the scatter of measurements of nasal transepithelial potentials in sick neonates (8) who had no evidence of epithelial injury, again suggesting that other factors are also involved.

Liquid junction potentials may have caused a bias of constant magnitude affecting the measured values of the TDP. The liquid junction potential arising from the $0.9 \%$ sodium chloride bridgeblood junction was measured in vitro, but that arising at the $0.9 \%$ sodium chloride $/ 0.75 \%$ agar-skin junction could not be measured. Thus, the overall effect of liquid junction potentials was not quantifiable but likely to be small. To avoid bias from different liquid junction potentials, future experiments should use electrolyte bridges of identical composition to be directly comparable with these results.

The mapping of TDP and its variability over the body surfaces enables selection of sites where the mean TDP is large in relation to the SD of the observations. Selection of these sites for measurement may show the greatest differences between control and experimental subjects when studying factors that influence TDP. Such observations may be of use to studies of biopotentials in the fields of psychology, physiology, and medicine.

\section{REFERENCES}

I. Edelberg R 1968 Biopotentials from the skin surface: the hydration effect. Ann NY Acad Sci 148:252-262

2. Sato K 1984 Differing luminal potential difference of cystic fibrosis and control sweat secretory coils in vitro. Am J Physiol 247:R649

3. Quinton PM 1983 Chloride impermeability in cystic fibrosis. Nature 301:421422

4. Hashimoto K, Gross BG, Lever WF 1965 The ultrastructure of the skin of human embryos I. The intraepidermal eccrine sweat duct. J Invest Dermatol 45:139-151

5. Harpin VA, Rutter N 1982 Sweating in preterm babies. J Pediatr 100(4):614618

6. Harpin VA, Rutter N 1982 Development of emotional sweating in the newborn infant. Arch Dis Child 57:691-695

7. Holbrook KA 1982 Neonatal Skin Structure and Function. Marcel Dekker Inc, New York, p 20

8. Gowen CW, Lawson EE, Gingras-Leatherman J, Gatzy JT, Boucher RC, Knowles MR 1986 Increased nasal potential difference and amiloride sensitivity in neonates with cystic fibrosis. J Pediatr 108(4):517-527 